\title{
Reactive Planning for Assistive Robots
}

\author{
Bevilacqua, P., Member, IEEE, Frego, M., Fontanelli, D. Member, IEEE, Palopoli, L., Member, IEEE
}

\begin{abstract}
We consider a vehicle consisting of a robotic walking assistant pushed by a user. The robot can guide the person along a path and suggest a velocity by various means. The vehicle moves in a crowded environment and can detect the different pedestrian in the surroundings. We propose a reactive planner that modifies the path in order to avoid the pedestrian in the surroundings. The algorithm relies on a very accurate model to predict the motion of each pedestrian, i.e. the Headed Social Force Model (HSFM). The possible trajectories for both the vehicle and the pedestrians are modelled as clothoid curves, which are efficient to manage from the numeric point of view and are very comfortable to follow for the user. Probabilistic techniques are used to account for the variability of the motion of each pedestrian. The path is efficient to generate, is collision free (up to a certain probability) and is comfortable to follow. Simulations and comparisons with a state of the art planner using real data as well as experiments are reported to prove the effectiveness of the method.
\end{abstract}

Index Terms-Assistive Robots, Reactive Planning, Motion Planning

\section{INTRODUCTION}

Service robots are increasingly used to help older adults in navigation tasks, to prolong their independent living and to keep them physically active. The techniques proposed in the paper target a large class of robotic devices that can be used for this purpose, even though we will make explicit reference to a specific intelligent robotic walker, the FriWalk developed in the context of the European project ACANTO [1] (see Figure 1). The FriWalk is a standard commercial walking aid endowed with sensing abilities to understand the surroundings, with communication abilities to connect to cloud services and with planning abilities to produce safe paths in the environment [2]. The specific problem considered here is the so called reactive planning, which is used when a senior user of the robotic walker encounters an unforeseen obstacle while following a planned trajectory (hereinafter global path, GP). In such cases as this, the reactive planner comes into play and generates a new local path (LP) that avoids the obstacle. In the following, the ensemble user+walker will be referred to as vehicle. The purpose of the reactive planner is to minimise the local deviations ensuring that the LP joins the GP shortly after the obstacle. Generally speaking, the planned path has to respect boundaries and dynamic constraints, which restrict the possible trajectories that can be taken. The occasional presence of obstacles, such as other human beings standing by or walking

P. Bevilacqua, M. Frego and L. Palopoli are with the Dipartimento di Ingegneria e Scienza dell'Informazione, University of Trento, Italy

D. Fontanelli is with the Dipartimento di Ingegneria Industriale, University of Trento, Italy

The research in this paper has received funding from the European Unions Horizon 2020 Research and Innovation Programme - Societal Challenge 1 (DG CONNECT/H) under grant agreement $n^{\circ} 643644$ "ACANTO - A CyberphysicAl social NeTwOrk using robot friends".

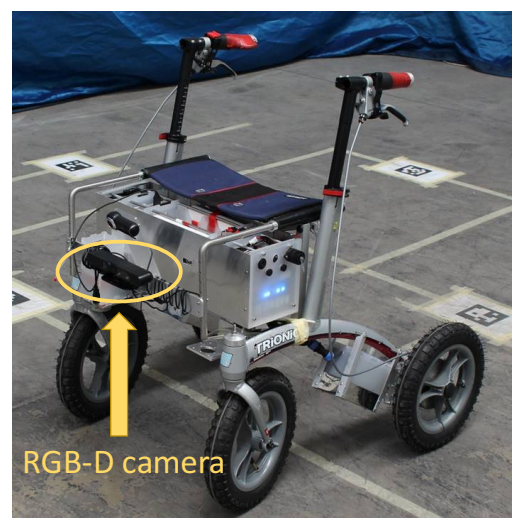

Fig. 1. A comparison between the proposed approach and the application of Risk-RRT for the dynamic re-planning.

in the neighbourhood of the vehicle, generates additional geometric and dynamic constraints. A few important assumptions underlie the design of the reactive planner. First, we assume the prior knowledge of an optimal GP (e.g., computed by a high level planner [2]) that avoids static obstacles [3]. Therefore, the reactive planner always has an available solution: stay on the GP, slow down or stop when dynamic obstacles come across and wait until they pass by. Second, the vehicle is equipped with a sensing system able to reveal obstacles and anomalous conditions in the surroundings (primarily, people walking in the proximity of the path as in [4]), for example an RGB-D camera as in the case of the FriWalk [5] (see Figure 1). Third, the trajectory re-planning has to be computed in real-time and using a lean hardware to reduce the cost.

Related work. Recently, different techniques have been developed to plan effective, collision free trajectories in the presence of dynamic and non-deterministic obstacles. In [6], uncertainties both on the robot state and on the dynamic obstacles are considered. The problem is formalised as a chance constrained optimisation, and an efficient, approximated solution is proposed. However, this approximation is valid for "small" robots and Gaussian distributions of the uncertainties. In [7], instead, the stochastic optimisation problem is solved by introducing the Partially Closed Loop Receding Horizon Control algorithm, while the uncertainty is kept in check using future data acquisitions. In [8], a velocity obstacle representation is used, hence the set of velocities causing a collision with an obstacle before a given time horizon is determined. Other solutions are based on the construction of a probabilistic occupancy map, and on the search of a safe path using improvements of classical path planning algorithms, as in the case of Probabilistic Roadmaps [9], or Risk-RRT [10]. The latter solution is based on the modelling of possible 
trajectories using a mixture of Gaussian Processes, and on the search of the solution using an adaptation of the RRT path planning algorithm [11] taking into account the probability of collision to determine the cost of the different plans. The techniques mentioned so far do not take into consideration the specific motion patterns of human beings in a crowded environment and, in general, require a high computation time. Another problem with these techniques is that they do not constrain the curvature of the generated path to be continuous (for instance, as shown below, Risk-RRT generates trajectories with piece-wise constant curvature). As a result the generated path could be jerky and uncomfortable.

More directly related to our approach are solutions based on the determination of families of trajectories usually followed by humans in a certain environment. Such trajectories can be modelled as Gaussian Processes [9], interactive Gaussian processes [12] or Hidden Markov Models [13]. The probabilistic information on the future evolution of the states of the dynamic obstacles is then used in different ways to find probabilistically safe trajectories. In this line of work, a large number of prior observations in a specific environment is used to fit the model parameters. Our efforts are toward solutions that are more generally applicable.

More accurate models describing the human motion are considered in a different thread of research papers [14], [15]. The idea is in these cases to use Montecarlo simulations to predict the future positions of the obstacles. This solution comes at the cost of a non-negligible computation time, which could potentially compromise the possibility of a real-time execution.

Our main goal in this work is to develop a computationally efficient reactive planner, which is particularly focused on avoiding collision with humans in crowded environments and which produces a comfortable path for the user. A correct understanding of how people move in a crowded environment is key to our work. The widely known Social Force Model (SFM) [16] assumes that a person is supposed to be able to move freely in any direction at any time, acting like a mass particle subjected to external forces. On the contrary, empirical evidence shows that, most of the time, pedestrians tend to move forward, i.e. their velocity vector is most often aligned with their heading, due to the biomechanics of humans. This phenomenon has been observed by several studies [17], [18], [19], which come to the conclusion that a nonholonomic model, e.g. unicycle-like or car-like models, may be more appropriate to describe human motion in many cases. The adoption of such models gives a nice interpretation of the mechanism underlying the formation of human trajectories, namely the minimisation of the derivative of the path curvature, the jerk [18]. In [20] the Headed Social Force Model (HSFM) is proposed to enhance the traditional SFM by explicitly accounting for the pedestrians' heading and thus retrieving the smoothness of the trajectories.

Paper Contribution. The Reactive Planner proposed in this paper uses the HSFM to predict the possible future motion of the obstacles (humans, in our context). Since the possible consequences of accidents are limited in our operational scenarios, we adopt a probabilistic formulation. Therefore, our reactive planner seeks the LP that avoids all the obstacles with an assigned probability minimising the deviation from the GP. Contrary to previous work [14], [15], our reactive planner is not based on run-time simulations. The key idea to adopt the HSFM as the baseline for the planner is to collect a large amount of data generated by humans (which can come from simulations or observations on the field). If we restrict to a relatively small time horizon, each generated trajectory can be closely approximated with clothoid curves [21], [22]. Clothoids have been found to be a good approximation of the motion of human trajectories [23] and can be efficiently manipulated in numeric algorithms. Each one of these curves is associated with a probability, associated with each possible choice of the pedestrian on her/his velocity and destination. The planner considers alternative paths (which are themselves clothoids) and for each of them computes the possible intersections with the pedestrian that may cause a collision. The use of these probabilities allows us to define a probabilistic performance index associated with the probability of a collision event. This way, it is possible to select a trajectory with small collision probabilities that minimises the waiting time and the distance from the GP.

The HSFM-based solution presented in this paper guarantees that the transition between the two paths is smooth up to the second derivative (the curvature), which makes it easy to track. Finally, the solution is efficient and can be implemented in real-time on lean hardware. In order to validate the approach, we show how the re-planning can be executed in a few milliseconds (on a standard machine).

The paper is organised as follows. In Section II, we present a detailed description of the adopted model of moving pedestrians. In Section III we illustrate how we formalise and solve the problem of re-planning in environments populated by humans, while Section IV assesses the validity of the finding through experimental validation. Finally, Section V provides some concluding remarks and proposes future research directions.

\section{Pedestrian Modelling}

The HSFM [20] is a recently introduced model to describe the motion of pedestrians in a social environment. In this model, the $i$-th individual is assimilated to a particle with mass $m_{i}$, whose position expressed in the world reference frame $\langle W\rangle$ is denoted by $\mathbf{r}_{i}=\left[x_{i}, y_{i}\right]^{\top}$. In order to model the pedestrians' heading, it is convenient to attach a body frame $\langle B\rangle$ to each individual, i.e. a reference frame centred at the pedestrian's position and whose $x$-axis is aligned with the pedestrian's forward direction of motion. Let $\mathbf{q}_{i}=\left[\theta_{i}, \omega_{i}\right]^{\top}$ be the vector containing the $i$-th pedestrian's heading and angular velocity are $\theta_{i}$ (angle between the $x$-axis of the body frame and that of the global reference frame) and $\omega_{i}=\dot{\theta}_{i}$, respectively. Denote by $\mathbf{v}_{i}^{B}=\left[v_{i}^{f}, v_{i}^{o}\right]^{\top}$ the velocity vector expressed in the body frame. The components $v_{i}^{f}$ and $v_{i}^{o}$ of vector $\mathbf{v}_{i}^{B}$ correspond to the projection of the velocity vector $\mathbf{v}_{i}$ along the forward direction and the orthogonal direction, respectively. Then, similarly to [24], the human locomotion model becomes

$$
\dot{\mathbf{r}}_{i}=\mathbf{R}_{o}\left(\theta_{i}\right) \mathbf{v}_{i}^{B}, \quad \dot{\mathbf{v}}_{i}^{B}=\frac{1}{m_{i}} \mathbf{u}_{i}^{B}, \text { and } \dot{\mathbf{q}}_{i}=\mathbf{A} \mathbf{q}_{i}+\mathbf{b}_{i} u_{i}^{\theta}
$$


where $\mathbf{R}_{o}\left(\theta_{i}\right)$ is the $2 \mathrm{D}$ rotation matrix of angle $\theta_{i}$,

$$
\mathbf{A}=\left[\begin{array}{cc}
0 & 1 \\
0 & 0
\end{array}\right], \quad \mathbf{b}_{i}=\left[\begin{array}{c}
0 \\
\frac{1}{I_{i}}
\end{array}\right]
$$

and $I_{i}$ denotes the moment of inertia of pedestrian $i$. In the model (1), the inputs are $\mathbf{u}_{i}^{B}=\left[u_{i}^{f}, u_{i}^{o}\right]^{\top}$, whose entries are the forces acting along the forward direction and the orthogonal direction, respectively, as well as the torque $u_{i}^{\theta}$ about the vertical axis. In this model, if we set $v_{i}^{o}(0)=0$ and $u_{i}^{o}(t)=0$, for all $t$, the dynamic unicycle model is recovered, hence the model features a nonholonomic behaviour [20].

As in the SFM [16], the HSFM model inputs $u_{i}^{f}, u_{i}^{o}$ and $u_{i}^{\theta}$ are computed on the basis of external forces. Two terms are considered: the first is $\mathbf{f}_{i}^{0}$ and accounts for the pedestrian's desire to move with a given velocity vector $\mathbf{v}_{i}^{d}$, i.e.

$$
\mathbf{f}_{i}^{0}=m_{i} \frac{\mathbf{v}_{i}^{d}-\mathbf{v}_{i}}{\tau_{i}}
$$

where $\tau_{i}>0$ is a parameter determining the rate of change of the velocity vector. The second term $\mathbf{f}_{i}^{e}$ is the sum of the forces generated by the environment, e.g. fixed obstacles, walls, furnitures, etc., and other pedestrians in the environments. Then, $u_{i}^{f}$ is given by the projection of $\mathbf{f}_{i}^{0}+\mathbf{f}_{i}^{e}$ along the pedestrian forward direction, $u_{i}^{o}$ has a dumped dynamic depending only on the external forces $\mathbf{f}_{i}^{e}$ scaled by the gain parameter $k^{o}>0$ and projected on the orthogonal to the pedestrian heading (hence, in open spaces the nonholonomic behaviour is recovered), while $u_{i}^{\theta}$ has again a second order dynamic depending on the constants $k^{\lambda}>0$ and $\alpha>1$ shaping its rate of convergence towards the desired heading [20].

\section{A. Approximation of the HSFM trajectories with clothoid curves}

The richness of the HSFM does not allow us to directly use it in our reactive planner for the computational cost of solving a set of differential equations on line. Moreover, the uncertainty about the model parameters of the actual person encountered and the fact that we can only detect a moving obstacle within a range of about 3 meters (sensing limit), justify a model simplification. In such a short space, we have observed that the trajectories produced by the HSFM can be approximated by the concatenation of only two clothoid arcs. As note above, this is in perfect accordance with the findings of Laumond et al. [23], [3]. It is worth noting that this is true if the moving pedestrian is not walking in a heavily populated environment, which will be an assumption in the rest of the paper. Our first step is to show how a clothoid spline can be found to approximate a given path generated by the HSFM.

Our idea is to approximate each trajectory generated by a configuration of a HSFM through two approximating clothoid arcs: the first one is a clothoid curve, whereas the second is a straight line (a special case of a clothoid). The construction requires to find the parameters of two clothoids, staring in $P_{0}$ and ending in $P_{2}$, that meet in the middle at point $P_{1}$ with curvature continuity, such that the second segment is a straight line (see Figure 2 for reference). A general clothoid curve is

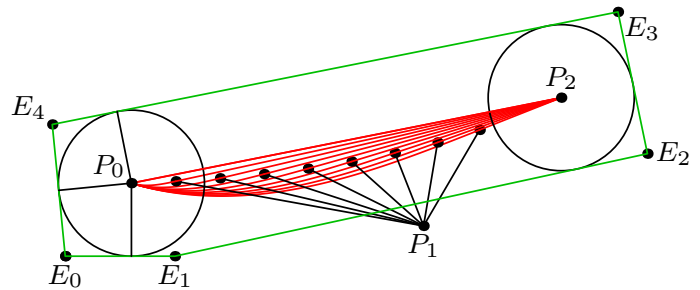

Fig. 2. Ten different two-segments clothoid splines that join with curvature continuity a starting point $P_{0}$ and reach a final (target) point $P_{2}$. The points labelled with $P_{1}$ are the connection points between first and second segment, respectively proper clothoid and straight line. The splines are modelled with ten different values of the percentage $p$ ranging from $0 \%$ to $100 \%$ with step $10 \%$. In green it is shown a bounding polygon $E_{0} E_{1} \ldots E_{4}$ that contains all the possible splines with a constant offset.

defined as [21]

$$
\begin{aligned}
x(s) & =x_{0}+s X_{0}\left(\kappa^{\prime} s^{2}, \kappa s, \beta\right), \\
y(s) & =y_{0}+s Y_{0}\left(\kappa^{\prime} s^{2}, \kappa s, \beta\right), \\
X_{n}(a, b, c) & =\int_{0}^{1} \eta^{n} \cos \left(\frac{a}{2} \eta^{2}+b \eta+c\right) \mathrm{d} \eta, \\
Y_{n}(a, b, c) & =\int_{0}^{1} \eta^{n} \sin \left(\frac{a}{2} \eta^{2}+b \eta+c\right) \mathrm{d} \eta,
\end{aligned}
$$

where $s$ is the curvilinear abscissa, $x_{0}, y_{0}$ are the coordinates of the starting point, $\beta$ is the initial angle, $\kappa$ is the initial curvature and $\kappa^{\prime}$ is the curvature change rate. $X_{n}$ and $Y_{n}$ in (5) are the Fresnel Generalised Integrals (FGI). The clothoid curve has the notable properties that the tangent is a quadratic polynomial and the curvature is a linear (affine) function of the arc length. Our method seeks the path that joins the two positions $P_{0}$ and $P_{2}$, respectively the current position of the sensed pedestrian and the hypothesised final position. At $P_{0}$ we measure the $x y$-position, that is, $P_{0}=\left(x_{0}, y_{0}\right)$, and an initial angle $\beta_{0}$. The second segment must be a straight line that meets the given final point $P_{2}=\left(x_{2}, y_{2}\right)$, hence the curvatures $\kappa^{\prime}$ and $\kappa$ must be zero. To model the different shapes of the resulting spline as in Figure 2, we use a tuning parameter $p \in(0,1)$ and define the length $L_{2}$ of the straight line as a percentage of the Euclidean distance between $P_{0}$ and $P_{2}$. In other words we set $L_{2}=p \cdot \operatorname{dist}\left(P_{0}, P_{2}\right)$. The tuning coefficient $p$ is assigned on the basis of the physical characteristics of the modelled pedestrian (more on this in the rest of this section). At the joining point $P_{1}$, which is unknown, we require $G^{2}$ geometric continuity up to the second derivative, which means that at the junction points the curvature is continuous. As a consequence, an additional constraint between the segments connecting $P_{i}$ to $P_{i+1}$ is defined and it requires that

$$
\begin{aligned}
H_{i, 0} & :=x_{i}+L_{i} X_{0}\left(\kappa_{i}^{\prime} L_{i}^{2}, \kappa_{i} L_{i}, \beta_{i}\right)-x_{i+1}, \\
H_{i, 1} & :=y_{i}+L_{i} Y_{0}\left(\kappa_{i}^{\prime} L_{i}^{2}, \kappa_{i} L_{i}, \beta_{i}\right)-y_{i+1}, \\
H_{i, 2} & :=\frac{1}{2} \kappa_{i}^{\prime} L_{i}^{2}+\kappa_{i} L_{i}+\beta_{i}-\beta_{i+1}, \\
H_{i, 3} & :=\kappa_{i}^{\prime} L_{i}+\kappa_{i}-\kappa_{i+1},
\end{aligned}
$$

must be equal to zero [3]. The subscript $i$ for $i=0,1,2$ refers to a condition relative to the point $P_{i}$, while the lengths of the two arcs are modelled with $L_{1}$ and $L_{2}$ respectively. Notice that 
$L_{i}$ runs from $i=1$ because the segments are one less than the points. Equations (6) and (7) ensure point-wise continuity, whereas (8) and (9) stand for the angle and curvature, $X_{0}$ and $Y_{0}$ are the functions defined in (5). This nonlinear system of equations is the $G^{2}$ Hermite Interpolation Problem with clothoids and is hard to solve in few milliseconds. However our particular form allows us to find a solution by means of the solution of the $G^{1}$ Hermite Interpolation Problem with clothoids [21]. In fact we can simplify some equations imposing the angles and curvatures in $P_{1}$ and $P_{2}$ to be equal (line segment). More precisely, this method solves all the previous equations but $H_{1,3}$ and yields the unknowns $L_{1}$, $\kappa^{\prime}$ and $\kappa_{0}$ as a function of $\beta_{1}$. The missing equation can be viewed as a function of the unknown parameter $\beta_{1}$ resulting from the $G^{1}$ solution: we can write it as

$$
h\left(\beta_{1}\right)=\kappa^{\prime}\left(\beta_{1}\right) L_{1}\left(\beta_{1}\right)+\kappa_{0}\left(\beta_{1}\right)=0 .
$$

In other words, with the $G^{1}$ solution, we can simplify the nonlinear system to a function of one variable $\beta_{1}$, as in (10), and hence using for example the Newton method with few iterations to solve it. In summary, the solution strategy to synthesise a path between $P_{0}$ and $P_{2}$ with two clothoids calls first the $G^{1}$ algorithm of [21], then finds, via the Newton method, the value of $\beta_{1}$ that solves (10). Experimental evidence shows that the problem at hand requires few iterations of the Newton method to converge in most of the cases.

\section{B. Fitting the clothoid spline to the HSFM parameters}

With the technique discussed above, we can approximate a short-horizon trajectory of the HSMF model with a simple two-segments spline of clothoids. The different shapes of this spline are created varying the percentage parameter $p$ previously mentioned, which models the behaviour of the pedestrian. The choice of $p$ depends from the parameters of the HSFM, which in turn model the pedestrian behaviour. In order to find this relation, we have simulated many trajectories between different pair of points $P_{0}$ and $P_{2}$ by changing all the HSFM parameters [20]. The result of this analysis is that the HSFM parameters that affect most the shape of the trajectory (and hence the choice of $p$ ) are: the reaction time of the pedestrian $\tau$, which is slower for young people and higher for more aged people; the parameter $k^{o}$ that models the orthogonal force, i.e. the step-aside trajectory; the values of the pedestrian heading dynamic behaviour $k^{\lambda}$ and $\alpha$, which determines if the curvature profile of the generated trajectory is loose or sharp. The metric adopted to compare the HSFM trajectory with the clothoid splines was the Root Mean Square Error (RMSE) based on the Euclidean distance. The adoption of this metric allowed us to construct the experimental map that associates to the $N$ different vectors $\left(\tau, k^{o}, k^{\lambda}, \alpha, \beta_{2}\right)$ the optimal percentage $p$ yielding the minimum RMSE. This map is a simple fitting function $\varphi$, which is a map $\varphi: \mathbb{R}^{5} \mapsto[0,1]$ such that $p=\varphi\left(\tau, k^{o}, k^{\lambda}, \alpha, \beta_{2}\right)$. The functional form of $\varphi$ is chosen as a multivariate polynomial of degree $d$, which has thus $n_{x}=\left(\begin{array}{c}n+d \\ d\end{array}\right)$ coefficients, collected in $\boldsymbol{x}$, for its monomials.
The fitting is performed by using least squares techniques based on QR decomposition, which means solving the least squares problem

$$
\min _{\boldsymbol{x}}\|\boldsymbol{A} \boldsymbol{x}-\boldsymbol{b}\|^{2}, \quad \boldsymbol{x} \in \mathbb{R}^{n_{x}}, \quad \boldsymbol{A} \in \mathbb{R}^{N \times n_{x}}, \quad \boldsymbol{b} \in \mathbb{R}^{N} .
$$

The matrix $\boldsymbol{A}$ is obtained evaluating for each row the monomials of the fitting polynomial on the particular configuration $\left(\tau, \kappa^{o}, \kappa^{\lambda}, \alpha, \beta_{2}\right)$, the corresponding right hand side $\boldsymbol{b}$ is the optimal value of $p$ related to the clothoids approximating that particular trajectory. The higher the degree $d$, (i.e., the dimension of $\boldsymbol{A}$ ), the harder the optimisation problem.

The QR decomposition of $\boldsymbol{A}$ produces a factorisation of the form $\boldsymbol{A}=\boldsymbol{Q R} \in \mathbb{R}^{N \times n_{x}}, \boldsymbol{Q} \in \mathbb{R}^{N \times n_{x}}, \boldsymbol{R} \in \mathbb{R}^{n_{x} \times n_{x}}$. The problem is then easily solved because

$$
\|\boldsymbol{A} \boldsymbol{x}-\boldsymbol{b}\|^{2}=\|\boldsymbol{Q} \boldsymbol{R} \boldsymbol{x}-\boldsymbol{b}\|^{2}=\left\|\boldsymbol{R} \boldsymbol{x}-\boldsymbol{Q}^{T} \boldsymbol{b}\right\|^{2}
$$

where we use the fact that $Q$ is an orthogonal matrix, e.g. $\boldsymbol{Q} \boldsymbol{Q}^{T}=\boldsymbol{I}$, and the Schwartz inequality of the last step becomes an equality under this hypothesis. Hence the problem is recast to the solution of the smaller linear system $\boldsymbol{R} \boldsymbol{x}-\boldsymbol{Q}^{T} \boldsymbol{b}$ which is also well conditioned. Producing this solution is fast because $\boldsymbol{R}$ is an upper triangular matrix. Since the problem is not ill conditioned, we can use a low degree $d$. By choosing $d=0$, we found for a large number of trajectories an approximate map $\varphi$ equal to a constant $p_{\text {opt }}=88 \%$. In plain words, this means that we can approximate a large number of pedestrian behaviour by a sequence of two clothoids with a fixed parameter $p$. We validated this result by executing random simulations for $N=160.000$ sets of parameters, and for 10 possible final destinations $P_{2}$ chosen at a distance of 3 metres from $P_{0}$ on an arc of circle for an angle between 0 and $\pi / 2$, and extended by symmetry arguments for the whole range $(-\pi / 2, \pi / 2)$. In all these cases the deviation of the HSFM trajectory with exact parameters from the curves with constant $p$ was below 0.5 [m], which is the approximate hindrance of the pedestrian. This value of $p$ corresponds to a good representative of average human behaviours, and the possible deviations of actual human types can be accounted for by increasing the volume of the obstacle when detecting a collision. We deem this approximation acceptable in the face of the drastic simplification in the computation time.

\section{Formalis ATION OF THE RE-PLANNING}

The result of the previous section can be summarised in the following terms: the short term motion of a pedestrian can be represented by a sequence of two clothoids characterised by a parameter $p$. Assuming that the final destination of the pedestrian is known, it is possible to single out a specific curve along which she/he is likely to move in a near future. In the discussion below we will make this assumption, while at the end of the section we will discuss how it can be removed.

Even when the path is known, in order to plan a trajectory, we also need to know how the pedestrian will move along the curve in time. Our assumption is that the pedestrian $H$ moves with a constant velocity $v_{0 \gamma}^{(h)}$ according to the linear ODE

$$
\dot{h}(t)=v_{0 \gamma}^{(h)}, \quad h(0)=h_{0}, \quad \Rightarrow \quad h(t)=h_{0}+v_{0 \gamma}^{(h)} t,
$$




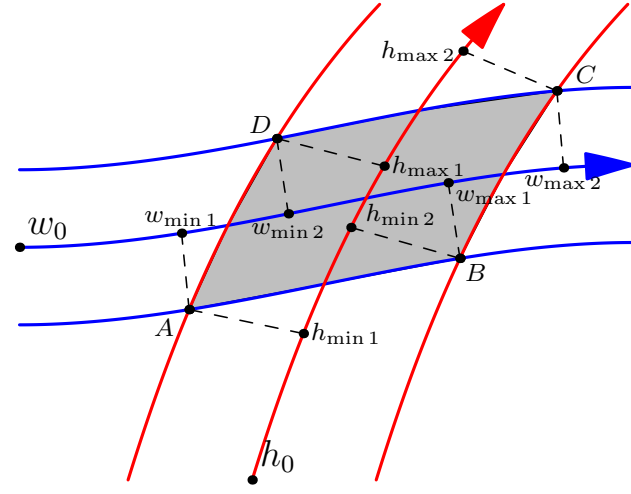

Fig. 3. Projection scheme of the geometric intersection points into the curvilinear abscissas of the walker and of the pedestrian. Those abscissas are then mapped to the corresponding time instants.

where $h(t)$ is the curvilinear abscissa of the obstacle over the generated clothoid path, i.e. the generic variable $s$ in (4). The constant velocity $v_{0 \gamma}^{(h)}$ is chosen randomly inside the interval $\left[v_{0 \min }^{(h)}, v_{0 \text { max }}^{(h)}\right]$ with known density $p_{\gamma}\left(v_{0 \gamma}^{(h)}\right)$ (which derives from past observations). Notice that the constant velocity assumption, quite popular in the field [25], [26], [27], is effective since the interaction between the vehicle and the pedestrian acts in a short amount of time. The constant velocity cumulative distribution function is $\mathbb{P}\left(v_{0 \gamma}^{(h)}<v\right)=$ $\int_{v_{0 \min }^{(h)}}^{v} p_{\gamma}\left(v_{0 \gamma}^{(h)}\right) \mathrm{d} v_{0 \gamma}^{(h)}$. Because of the pedestrian hindrance, the $\operatorname{space}$ around its centre of mass is described with a conservative offset $\pm \delta_{h}$ around the abscissa $h(t)$, hence the obstacle occupies the interval $\left[h(t)-\delta_{h}, h(t)+\delta_{h}\right]$ at time $t$. We model the walker vehicle $W$ in the same manner of the obstacle, with hindrance specified by $\delta_{w}{ }^{1}$. Its curvilinear abscissa is identified with the variable $w$ (see Figure 3). Each of the two agents $W$ and $H$, moves on a sequence of smoothly joined clothoids. The curve followed by the pedestrian is the spline made up of two segments (see Section II), while for the vehicle it is the candidate path. To handle the two paths and speed-up the computation of the possible intersection, we decompose each of the path into a sequence of triangles organised with the tree structure discussed in [3]. To model the physical encumbrance given by $\delta_{w}$ (respectively $\delta_{h}$ for the pedestrian), the spline has two parallel curves at the left and at the right (see Figure 3). We call a clothoid tunnel the clothoid spline and its two offset curves.

As a final remark, we will assume below, without loss of generality, that the velocity of the vehicle $W$ is constant along the planned path and that it is a decision variable.

\section{A. The velocity diagram}

We now discuss a tool (the velocity diagram), which can be used to detect collisions and to select the optimal velocity of the vehicle $W$.

In a standard intersection event, the routine for finding the collision considering the encumbrance will return a sequence of 4 points $\{A, B, C, D\}$ that are the vertexes of a generalised

\footnotetext{
${ }^{1}$ As observed above, a conservative estimate of the hindrance can include the possible uncertainty introduced by a constant choice of the parameter $p$.
}

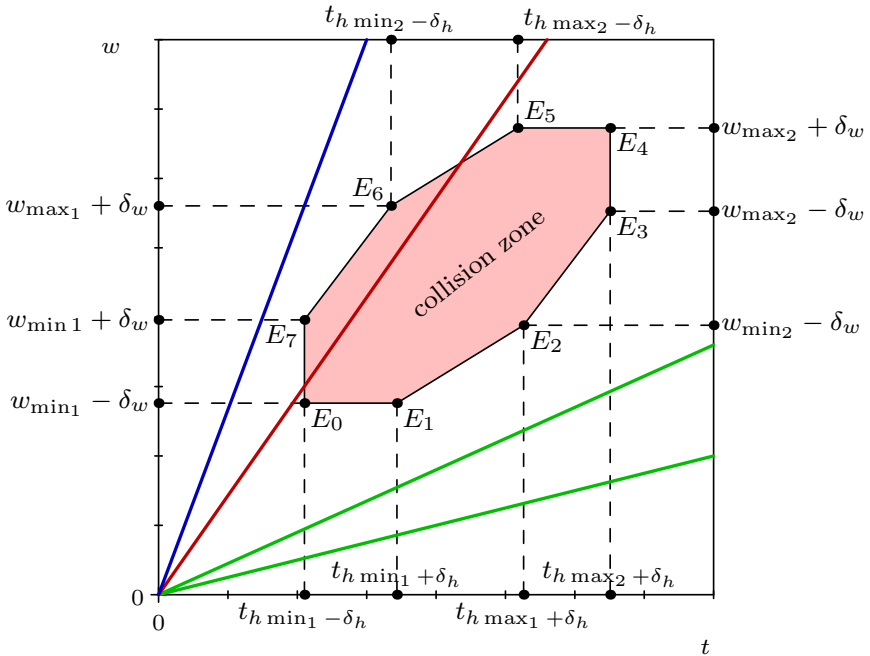

Fig. 4. The velocity diagram for a single intersection of trajectories: the red area represents the collision zone in terms of space and time. Green lines are walker's velocities that follow the pedestrian, red lines are walker's velocities that will cause a collision, and blue lines are velocity that allow the walker to overtake the obstacle.

quadrilateral, as depicted in Figure 3. It is possible to find situation in which the collision is not described by 4 point. For instance, when trajectories are almost tangent, a smaller number of points points will be found. However, these are simply degenerate cases in which some of the points of the generalised quadrilateral coincide. With the four geometric intersection points, we have to compute the curvilinear abscissas of the entry and exit points of the collision zone. They are called $w_{\min 1}$ and $w_{\max 2}$ for the walker and $h_{\min 1}, h_{\max 2}$ for the pedestrian (see Figure 3). To find those points, we applied again an effective Newton method that ends in few iterations.

Because of the agents hindrance, it is not enough to simply consider those coordinates. Therefore we call $w_{\min }=$ $w_{\min 1}-\delta_{w}, w_{\max }=w_{\max 2}+\delta_{w}$ and for the pedestrian $h_{\min }=h_{\min 1}-\delta_{h}, h_{\max }=h_{\max 2}+\delta_{h}$. From the curvilinear abscissas computing the travelling time is also an easy step, since by hypothesis both velocities are constant. Those time instants are called accordingly to the name of the abscissas respectively $t_{w \min }, t_{w \max }$, and the same for the obstacle but with subscript $h$. It is convenient to name the time intervals as $\Delta T_{h}:=\left[t_{h \min }, t_{h \max }\right]$ and $\Delta T_{w}:=\left[t_{w \min }, t_{w \max }\right]$. A collision happens if and only if the intersection $\Delta T_{h} \cap \Delta T_{w} \neq \emptyset$, i.e. the vehicle and the pedestrian are in the same region at the same time. Those quantities lead naturally to a spacetime representation, dubbed velocity diagram, in which the horizontal axis represents the time and the vertical axis the curvilinear abscissa $w(t)$ of the walker for a specified path. The velocities, being constant, are thus straight lines from the origin. More interestingly, the collision zone in space and time can be approximated with an octagon, as in Figure 4. The function $t_{s}=\Xi\left(v_{0 \gamma}^{(h)}, v_{0}^{(w)}\right)$ returns the minimum time required to wait to avoid the collision with fixed $v_{0}^{(w)}$. This is clearly zero if $\Delta T_{h} \cap \Delta T_{w}=\emptyset$. After the amount of time $t_{s}$, the vehicle can move with velocity $v_{0}^{(w)}$ and be sure to 
avoid the pedestrian. It has to be noted that the function $\Xi(\cdot)$ is solved by computing intersections between segments on the velocity diagram, hence computationally very light. Moreover, the constant velocity assumption on $v_{0}^{(w)}$ has been selected for simplicity and for the particular application at hand, since the user cannot be accelerated. Nevertheless, in a more general framework, time varying velocity profiles satisfying velocity constraints can be selected.

The previous graphical solution computes the stop time $t_{s}$ for a given $v_{0}^{(w)}$ and $v_{0 \gamma}^{(h)}$, where the latter is only known statistically. Therefore, we define the following cost index

$$
J_{t}=\int_{v_{0 \min }^{(h)}}^{v_{0 \max }^{(h)}} \Xi\left(v_{0 \gamma}^{(h)}, v_{0}^{(w)}\right) p_{\gamma}\left(v_{0 \gamma}^{(h)}\right) \mathrm{d} v_{0 \gamma}^{(h)},
$$

which somehow represents the average minimum time the vehicle has to wait to avoid the collision and it is a function of the chosen velocity $v_{0}^{(w)}$. Notice that a change in $v_{0 \gamma}^{(h)}$, corresponds to a translation and a scale of the polygon in Figure 4 . The velocity $v_{0}^{(w)}$ of the vehicle is then selected from a discrete set of values contained in $\left[v_{0}^{(w)}{ }_{\min }, v_{0}^{(w)}{ }_{\max }\right]$ such that (12) is minimised. Since in most of the cases the value of $\Xi(\cdot)$ will be zero, there will be multiple minima, say $V_{w}$. Hence, the selected $v_{0}^{(w)}$ will be the closest to a desired comfortable velocity $v_{d}^{(w)}$, i.e.

$$
J_{v}=\underset{v \in V_{w}}{\arg \min }\left|v-v_{d}^{(w)}\right| .
$$

The depicted algorithm determines the optimal $v_{0}^{(w)}$ in the sense of (12) and (13) along the selected path and assuming the pedestrian moves from its actual position $P_{0}$ to a well defined desired position $P_{2}$. If it is possible to modulate the velocity, we can accelerate the vehicle in order to overtake the obstacle (blue line in Figure 4), if we do not have enough escaping velocity it is possible to slow down or stop and let the obstacle pass (green lines in Figure 4). All the described computations are performed graphically in the velocity diagram and a solution always exists: in the worst case, the vehicle stops. However, if for the problem at hand the stop time $t_{s}$ is too high, the probability of having a collision (related to $J_{t}$ ) is too high or if the performance are too poor (a too high cost for $J_{v}$ ), a local re-planning is needed, which is the purpose of the next section.

\section{B. Releasing the assumptions}

In the previous section, we made the important assumption that the final point $P_{2}$ of the pedestrian is known. We can release this assumption introducing an additional random variable for $P_{2}$. Different papers in the literature give suggestions on the possible probability distributions for $P_{2}$ accounting for the social conventions [25], [28]. The analytical computation based on the velocity diagram discussed above can be repeated as a function of $P_{2}$, producing a re-formulation of the performance index in (12) in which the position of $P_{2}$ appears as an additional random variable $p_{2}$ to integrate on, and the distribution $p_{\gamma}\left(v_{0 \gamma}^{(h)}\right)$ is replaced by the joint distribution of $p_{2}$ and $v_{0 \gamma}^{(h)}$. As far as $p_{2}$ is assumed independent from $v_{0 \gamma}^{(h)}$

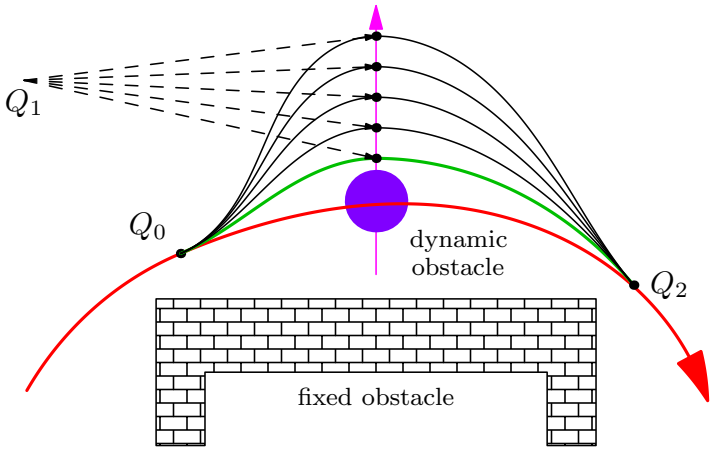

Fig. 5. A sketch of the local re-planning method, in red the global trajectory that is no more feasible because of the obstacle (purple circle). In green the optimal escaping manoeuvre, in black feasible candidates for different choices of $Q_{1}$ located deterministically aside from the obstacle trajectory.

the computation of the integral is straightforward. In the same way, it is possible to deal with a possible variability of the velocity of $W$. Indeed, while we can "suggest" a possible velocity to the user in different ways (e.g., through haptic devices or a GUI), it is not guaranteed that s/he will closely follow the suggestion, hence random variations around our selected velocity are a possibility.

\section{Local Re-planning}

In the same way as proposed by different authors [29] and in our previous work [30], our strategy for trajectory replanning is based on a decomposition of the problem into dynamic planning, in which the velocity profile on the path are computed as explained in the previous section, and geometric planning, which is the purpose of this section. When a replanning is requested, the algorithm selects a point $Q_{0}$ on GP with position $\left(x_{0}, y_{0}\right)$, angle $\beta_{0}$ and curvature $\kappa_{0}$ and a point $Q_{2}$ again on GP, with position $\left(x_{2}, y_{2}\right)$, angle $\beta_{2}$ and curvature $\kappa_{2}$; the re-planned trajectory will depart from $Q_{0}$ and will rejoin it at $Q_{2}$. The algorithm seeks a new point $Q_{1}$ in the proximity of the obstacle to pivot on to find the best trajectory (see Figure 5). The connecting curve is a spline of clothoid curves [21] that exhibits $C^{2}$ continuity with respect to the GP, and that is very fast to compute. The different choices of point $Q_{1}$ can be explored via a deterministic search (as herein done) or by using stochastic methods. In most of the reasonable application scenarios, the method reliably produces a solution. In the extreme cases in which it should not work, its efficiency leaves time to slow down the vehicle and back off to different emergency solutions, e.g. stop on the spot. In principle, the algorithm presented below operates with any pair of entry and exit points $Q_{0}, Q_{2}$ on the GP. An obvious requirement is that $Q_{0}$ and $Q_{2}$ be located before and after the obstacle. The low computational cost of the algorithm allows us to test different possible choices or, again, back off to an emergency strategy if the spline identified by the algorithm fails to satisfy the geometric or the dynamic constraints. However, the application of reasonable heuristics on the selection of $Q_{0}$ and $Q_{2}$ limits the occurrence of this anomaly. It is useful to observe that if the obstacle is very close to the vehicle, the back off solutions are likely to be adopted. An intuitive and straightforward way 

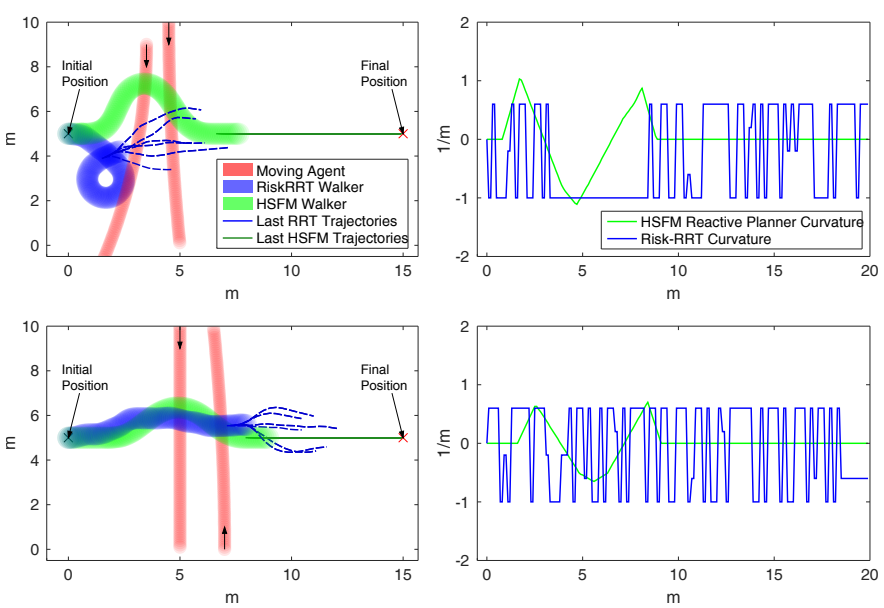

Fig. 6. A comparison between the proposed approach and the application of Risk-RRT for the dynamic re-planning.

to produce the points $Q_{0}$ and $Q_{2}$ is to identify them as the current position and at a distance which is reasonably far from the obstacle, respectively. Once a path is found, the optimal vehicle velocity $v_{0}^{(w)}$ minimising $J_{t}$ and $J_{v}$ in (12) and (13), respectively, is computed.

To compute the reactive re-planning we require two functions that solve the $G^{1}$ and $G^{2}$ Hermite Interpolation Problem for clothoid curves (HIP). The $G^{1}$ problem has been solved efficiently in [21], whereas the solution to the $G^{2}$ problem, in our case, asks for the solution of (6)-(9) for 3 arcs, $i=0,1,2,3$. The numeric solution algorithm adopted handles the system of equations quite efficiently, as reported next.

\section{EXPERIMENT WITH THE WALKER}

Our reactive-planning has been validated in two ways: a set of software simulations, where the dynamic obstacles were generated using the two clothoid model discussed in Section II according to the statistical data of recorded real pedestrians by ETH [31]; with a direct implementation on our robotic walker FriWalk. In this section we describe briefly the results obtained.

\section{A. Software simulations}

We have tested two situations where a pair of pedestrians cross the walker's trajectory, in one case they walk both in the same direction, in the other they come from opposite sides, see Figure 6 (left). In green it is depicted the trajectory generated by our reactive planner with the method herein discussed and in blue the solution provided by the Risk-RRT algorithm [10]. In the first test case (Figure 6, top left), our method produces a smooth deviation from the GP and reconnects to it after the obstacle avoidance. The approach based on Risk-RRT produces an unnatural loop and then reconnects to the GP. In the second case, both methods give similar natural solution (Figure 6, bottom left). However, when the curvature profile of the trajectories is considered, the one obtained by Risk-RRT (blue line in Figure 6, right) exhibits a piecewise constant behaviour with frequent jumps to three possible values: this is an undesirable property for the tracking system and also for

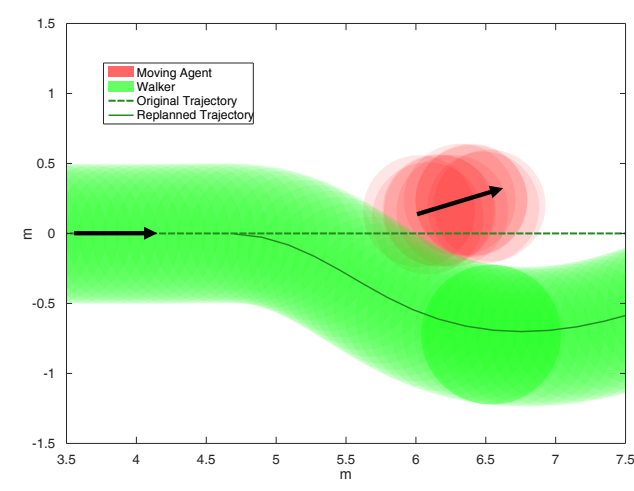

Fig. 7. Experimental re-planning example with the FriWalk.

the comfort of the user. In fact curvature discontinuities or at least sharp variations induce high spikes in the jerk profile. On the other hand, our method exploits the property that clothoids have linear curvature w.r.t. the arc length, resulting thus in a regular piecewise linear curve (green line in Figure 6, right).

\section{B. Walker implementation}

We have conducted some real experiments in the lobby hall of the University of Trento by implementing on the FriWalk the presented Reactive Planner. The results are in accordance with the computer simulations described so far. The computational times on the on board hardware (an Intel I7 5557 Nuc with 8GB of DDR3 RAM, used for the planner here presented and the landmark detection, the guidance algorithm and the GUI of the FriWalk [4], and a 1GHz ARM CortexA8 Beaglebone Black with 512MB of DDR3 RAM, used for data processing of sensors and actuators, and for the localisation algorithms running on the FriWalk [32]) show that it is possible to employ the proposed Reactive Planner for real time applications. In fact we registered a mean execution time of 3 [ms] for a standard escaping manoeuvre with 5 candidates $Q_{1}$ points (as depicted in Figure 5), the minimum time was about 1 [ms] and the maximum 11 [ms]. The acquisition of the moving obstacles was performed using an Asus Xtion Pro RGB-D sensor, with sampling time of 100 [ms], while the replanning module was executed with a period of 300 [ms]. Figure 7 depicts the results of one experiment: a human, walking in the same direction, enters the field of view of the FriWalk that determines the unfeasibility of the original path (dashed green line). Thus a new safe trajectory (solid green line) is generated by the reactive re-planner.

\section{Conclusions}

In this paper, we have considered a vehicle consisting of a robotic walker pushed by a human. The robot is endowed with sensing devices to detect the presence of obstacles in the surrounding, with guidance mechanisms to steer the user along a path and to suggest a velocity. The specific problem considered here is how to modify a planned path in order to avoid pedestrians when the vehicle moves in a crowded space. The key idea of the paper is to adopt the HSFM to predict 
the path followed by the pedestrians. We have shown how to translate such paths into pieces of clothoid curves, which are comfortable to follow and efficient to treat numerically. The use of these curves is at the heart of our planning solution. The uncertainty on the velocity followed by the pedestrians, on the points that s/he wants to reach and on the possible deviations of the user from the suggested speed are modelled as stochastic variables. Therefore, it is possible to define a probabilistic performance index and seek the velocity profile that minimises it for each candidate path. Different candidate paths are considered and the one that minimises both the performance index and the deviation from the global plan is selected.

Several issues remain open for future investigations. The most important is to consider the mutual interaction between several pedestrians and the vehicle and the pedestrians. Indeed, if social interactions are considered, the final position and the velocity random variables are obviously dependent and different computation approaches have to be developed. Nonetheless, for simple scenarios in which the vehicle interacts with one or two pedestrians every time, the use of independent variables apparently delivers a good performance at a low computational cost.

\section{REFERENCES}

[1] "ACANTO: A CyberphysicAl social NeTwOrk using robot friends," http://www.ict-acanto.eu/acanto, February 2015, EU Project.

[2] A. Colombo, D. Fontanelli, A. Legay, L. Palopoli, and S. Sedwards, "Efficient customisable dynamic motion planning for assistive robots in complex human environments," Journal of Ambient Intelligence and Smart Environments, vol. 7, no. 5, pp. 617-633, 2015.

[3] P. Bevilacqua, M. Frego, E. Bertolazzi, D. Fontanelli, L. Palopoli, and F. Biral, "Path planning maximising human comfort for assistive robots," in 2016 IEEE Conference on Control Applications (CCA). IEEE, 2016, pp. 1421-1427.

[4] L. Palopoli, A. Argyros, J. Birchbauer, A. Colombo, D. Fontanelli et al., "Navigation Assistance and Guidance of Older Adults across Complex Public Spaces: the DALi Approach," Intelligent Service Robotics, vol. 8, no. 2, pp. 77-92, 2015.

[5] P. Panteleris and A. A. Argyros, "Vision-Based SLAM and Moving Objects Tracking for the Perceptual Support of a Smart Walker Platform," in ECCV Workshops (3), 2014, pp. 407-423.

[6] N. E. Du Toit and J. W. Burdick, "Probabilistic collision checking with chance constraints," IEEE Transactions on Robotics, vol. 27, no. 4, pp. 809-815, 2011.

[7] _ , "Robot motion planning in dynamic, uncertain environments," IEEE Transactions on Robotics, vol. 28, no. 1, pp. 101-115, 2012.

[8] F. Large, D. Vasquez, T. Fraichard, and C. Laugier, "Avoiding cars and pedestrians using velocity obstacles and motion prediction," in Intelligent Vehicles Symposium, 2004 IEEE. IEEE, 2004, pp. 375-379.

[9] A. Alempijevic, R. Fitch, and N. Kirchner, "Bootstrapping navigation and path planning using human positional traces," in Robotics and Automation (ICRA), 2013 IEEE International Conference on. IEEE, 2013, pp. 1242-1247.

[10] C. Fulgenzi, C. Tay, A. Spalanzani, and C. Laugier, "Probabilistic navigation in dynamic environment using rapidly-exploring random trees and gaussian processes," in Intelligent Robots and Systems, 2008. IROS 2008. IEEE/RSJ International Conference on. IEEE, 2008, pp. 10561062.

[11] S. M. LaValle and J. J. Kuffner Jr, "Randomized kinodynamic planning," The International Journal of Robotics Research, vol. 20, no. 5, pp. 378400, 2001

[12] P. Trautman, J. Ma, R. M. Murray, and A. Krause, "Robot navigation in dense human crowds: Statistical models and experimental studies of humanrobot cooperation," The International Journal of Robotics Research, vol. 34, no. 3, pp. 335-356, 2015.

[13] Q. Zhu, "Hidden markov model for dynamic obstacle avoidance of mobile robot navigation," IEEE Transactions on Robotics and Automation, vol. 7, no. 3, pp. 390-397, 1991 .
[14] D. Althoff, J. J. Kuffner, D. Wollherr, and M. Buss, "Safety assessment of robot trajectories for navigation in uncertain and dynamic environments," Autonomous Robots, vol. 32, no. 3, pp. 285-302, Apr 2012.

[15] A. Colombo, D. Fontanelli, D. Gandhi, A. DeAngeli, L. Palopoli, S. Sedwards, and A. Legay, "Behavioural Templates Improve Robot Motion Planning with Social Force Model in Human Environments," in Proc. IEEE Int. Conf. on Emerging Technologies \& Factory Automation (ETFA). Cagliari, Italy: IEEE, 10-13 Sep. 2013, pp. 1-6.

[16] D. Helbing, I. Farkas, and T. Vicsek, "Simulating dynamical features of escape panic," Nature, vol. 407, pp. 487-490, September 2000.

[17] G. Arechavaleta, J.-P. Laumond, H. Hicheur, and A. Berthoz, "On the nonholonomic nature of human locomotion," Autonomous Robots, vol. 25 , no. $1-2$, pp. $25-35,2008$.

[18] _ _An optimality principle governing human walking," IEEE Transactions on Robotics, vol. 24, no. 1, pp. 5-14, Feb 2008.

[19] G. Ferrer and A. Sanfeliu, "Proactive kinodynamic planning using the extended social force model and human motion prediction in urban environments," in 2014 IEEE/RSJ International Conference on Intelligent Robots and Systems. IEEE, 2014, pp. 1730-1735.

[20] F. Farina, D. Fontanelli, A. Garulli, A. Giannitrapani, and D. Prattichizzo, "Walking ahead: The headed social force model," PLOS ONE, vol. 12, no. 1, pp. 1-23, 012017.

[21] E. Bertolazzi and M. Frego, "G1 fitting with clothoids," Mathematical Methods in the Applied Sciences, vol. 38, no. 5, pp. 881-897, 2015. [Online]. Available: http://dx.doi.org/10.1002/mma.3114

[22] M. Frego, E. Bertolazzi, F. Biral, D. Fontanelli, and L. Palopoli, "Semi-analytical minimum time solutions with velocity constraints for trajectory following of vehicles," Automatica, vol. 86, pp. 18 - 28, 2017.

[23] G. Arechavaleta, J.-P. Laumond, H. Hicheur, and A. Berthoz, "An Optimality Principle Governing Human Walking," IEEE Transactions on Robotics, vol. 24, no. 1, pp. 5-14, Feb 2008.

[24] K. Mombaur, A. Truong, and J.-P. Laumond, "From human to humanoid locomotion - an inverse optimal control approach," Autonomous robots, vol. 28, no. 3, pp. 369-383, 2010.

[25] M. Luber, J. A. Stork, G. D. Tipaldi, and K. O. Arras, "People tracking with human motion predictions from social forces," in Proc. IEEE International Conference on Robotics and Automation (ICRA'10), Anchorage, USA, 2010.

[26] Q. Zhu, "Hidden Markov model for dynamic obstacle avoidance of mobile robot navigation," IEEE Transactions on Robotics and Automation, vol. 7, no. 3, pp. 390-397, Jun 1991.

[27] N. Du Toit and J. Burdick, "Robot motion planning in dynamic, uncertain environments," IEEE Transactions on Robotics, vol. 28, no. 1, pp. 101-115, Feb 2012

[28] M. Bennewitz, W. Burgard, G. Cielniak, and S. Thrun, "Learning motion patterns of people for compliant robot motion," The International Journal of Robotics Research, vol. 24, no. 1, pp. 31-48, 2005.

[29] E. Velenis and P. Tsiotras, "Minimum-time travel for a vehicle with acceleration limits: Theoretical analysis and receding-horizon implementation," Journal of Optimization Theory and Applications, vol. 138, no. 2, pp. 275-296, 2008.

[30] M. Frego, P. Bevilacqua, E. Bertolazzi, F. Biral, D. Fontanelli, and L. Palopoli, "Trajectory planning for car-like vehicles: A modular approach," in 2016 IEEE 55th Conference on Decision and Control (CDC), Dec 2016, pp. 203-209.

[31] S. Pellegrini, A. Ess, K. Schindler, and L. van Gool, "You'll never walk alone: Modeling social behavior for multi-target tracking," in 2009 IEEE 12th International Conference on Computer Vision, Sept. 2009, pp. 261268, http://www.vision.ee.ethz.ch/datasets/index.en.html.

[32] P. Nazemzadeh, F. Moro, D. Fontanelli, D. Macii, and L. Palopoli, "Indoor Positioning of a Robotic Walking Assistant for Large Public Environments," IEEE Trans. on Instrumentation and Measurement, vol. 64, no. 11, pp. 2965-2976, Nov 2015. 